\title{
RADIATION FIELDS ON ASYMPTOTICALLY EUCLIDEAN MANIFOLDS
}

\author{
ANTÔNIO SÁ BARRETO
}

\begin{abstract}
F.G. Friedlander introduced the notion of radiation fields for asymptotically Euclidean manifolds. Here we answer some of the questions he proposed and apply the results to give a unitary translation representation of the wave group, and to obtain the scattering matrix for such manifolds. We also obtain a support theorem for the radiation fields.
\end{abstract}

\section{Introduction and Statement of the Results}

Friedlander $[1,2]$ introduced the forward radiation fields for asymptotically Euclidean manifolds as the limit, as times goes to infinity, of the forward fundamental solution of the wave operator along certain light rays. By reversing time one defines the backward radiation field. We will show that this leads to a unitary translation representation of the wave group and a dynamical definition of the scattering matrix. Similar results for asymptotically hyperbolic manifolds have been established in [13].

A smooth compact manifold $X$ with boundary, $\partial X$, is called asymptotically Euclidean, or scattering manifold see [9], when it is equipped with a Riemannian metric $g$, which is smooth in the interior of $X$, denoted by $\stackrel{\circ}{X}$, and can be written as

$$
g=\frac{d x^{2}}{x^{4}}+\frac{\mathcal{H}}{x^{2}}
$$

near $\partial X$. Here $x \in C^{\infty}(X)$ is a smooth defining function of $\partial X$ and $\mathcal{H}$ is a symmetric tensor which restricts to a smooth Riemannian metric $h_{0}$ on $\partial X$. As observed in $[8,9]$, once (1.1) is known to exist, it determines $x$ up to terms vanishing to second order at $\partial X$, and hence it determines $h_{0}=\left.\mathcal{H}\right|_{\partial X}$. We will denote the dimension of $X$ by $n$, and therefore $\partial X$ has dimension $n-1$.

The Euclidean space $\mathbb{R}^{n}$ can be viewed as such a manifold by compactification. As in [9], let

$$
\mathbb{S}_{+}^{n}=\left\{y \in \mathbb{R}^{n+1}:|y|=1, \quad y_{1}>0\right\}
$$

denote the upper hemisphere of the unit sphere in $\mathbb{R}^{n+1}$. Its boundary is $\partial \mathbb{S}_{+}^{n}=\mathbb{S}^{n-1}$. Let

$$
\begin{gathered}
S P: \mathbb{R}^{n} \longrightarrow \mathbb{S}_{+}^{n} \\
z \longmapsto\left(\frac{1}{\left(1+|z|^{2}\right)^{\frac{1}{2}}}, \frac{z}{\left(1+|z|^{2}\right)^{\frac{1}{2}}}\right) .
\end{gathered}
$$

The Euclidean metric when pushed forward to $\mathbb{S}_{+}^{n}$ has the form (1.1). Moreover, as observed in [10], any perturbation of the Euclidean metric which behaves like

$$
g_{i j}=\delta_{i j}+\frac{1}{|z|^{2}} h_{i j}\left(\frac{1}{|z|}, \frac{z}{|z|}\right), \quad \text { as }|z| \rightarrow \infty
$$

satisfies (1.1) when pushed forward to $\mathbb{S}_{+}^{n}$.

In odd dimensional Euclidean spaces, the forward radiation field is the modified Radon transform of Lax and Phillips, see Proposition 4.20 of [1] or Theorem 2.4 of chapter 4 of [7]. Therefore the radiation fields can be viewed as a generalization of such transform to asymptotically Euclidean manifolds. The methods developed here also provide new proofs, although rather difficult ones, of well known results 
about the Radon transform. In particular, Theorem 4.2 can be viewed as a generalization of the well known support theorem for Radon transform due to Helgason [4].

The methods we use to establish the relationship between the radiation fields and the scattering matrix are adaptations of the techniques of $[1,2]$ and the construction of the resolvent of the Laplacian due to Hassell and Vasy [3].

It is shown in [6] that if $g$ satisfies (1.1) there exists $\epsilon>0$ and a product structure $X \sim \partial X \times[0, \epsilon)$ in which

$$
g=\frac{d x^{2}}{x^{4}}+\frac{h(x, y, d y)}{x^{2}} .
$$

These are the equivalent of boundary normal coordinates on a compact manifold with boundary.

We will fix such a decomposition and $x \in C^{\infty}(X)$ will be as in (1.2). Let $\Delta$ be the (positive) Laplace operator with respect to the metric $g$.

It was shown in [9] that given $f \in C^{\infty}(\partial X)$ and $\lambda \in \mathbb{R}, \lambda \neq 0$, there exists a $u \in C^{\infty}(\stackrel{\circ}{X})$ satisfying

$$
\begin{gathered}
\left(\Delta-\lambda^{2}\right) u=0 \text { in } \stackrel{\circ}{X} \\
u=x^{\frac{n-1}{2}} e^{i \frac{\lambda}{x}} F+x^{\frac{n-1}{2}} e^{-i \frac{\lambda}{x}} G, \quad F, G \in C^{\infty}(\bar{X}),\left.\quad F\right|_{\partial X}=f .
\end{gathered}
$$

This leads to the stationary definition of the scattering matrix at energy $\lambda$. Melrose defined it in [9] as the operator

$$
\begin{gathered}
A(\lambda): C^{\infty}(\partial X) \longrightarrow C^{\infty}(\partial X) \\
\left.f \longmapsto G\right|_{\partial X} .
\end{gathered}
$$

Melrose and Zworski showed in [10] that $A(\lambda)$ is a classical Fourier integral operator of order zero associated to the geodesic flow at time $\pi$ given by the metric $h_{0}$ on $\partial X$.

Here, as in $[1,2,13]$, we will use the wave equation to define the radiation fields and arrive at an equivalent definition of the scattering matrix.

Friedlander proved

Theorem 1.1. [2] For $f_{1}, f_{2} \in C_{0}^{\infty}(X)$ compactly supported in the interior of $X$, let

$$
\begin{gathered}
u(t, z) \in C^{\infty}\left(\mathbb{R}^{+} \times X\right) \text { satisfy } \\
\left(D_{t}^{2}-\Delta\right) u(t, z)=0, \text { on } \mathbb{R} \times \stackrel{\circ}{X}, \\
u(0, z)=f_{1}(z), \quad D_{t} u(0, z)=f_{2}(z) .
\end{gathered}
$$

Let $z=(x, y) \in(0, \epsilon) \times \partial X$ be local coordinates near $\partial X$ in which (1.2) hold. Let $H(t)=1$ for $t>0$ and $H(t)=0$ otherwise, denote the Heaviside function. Then there exist $w_{k} \in C^{\infty}(\mathbb{R} \times \partial X)$, such that

$$
x^{-\frac{n-1}{2}}(H u)\left(s+\frac{1}{x}, x, y\right) \sim \sum_{k=0}^{\infty} x^{k} w_{k}(s, y), \quad \text { as } x \rightarrow 0 .
$$

In particular,

$$
\left.x^{-\frac{n-1}{2}}(H u)\left(s+\frac{1}{x}, x, y\right)\right|_{x=0}=w_{0}(s, y),
$$

is well defined.

As any two boundary defining functions satisfying (1.2) agree to second order at $\partial X, w_{0}(s, y)$ is independent of the choice of $x$. 
Consider the forward fundamental solution of the wave operator $U\left(t, z, z^{\prime}\right)$ which satisfies

$$
\begin{gathered}
\left(D_{t}^{2}-\Delta\right) U\left(t, z, z^{\prime}\right)=\delta(t) \delta\left(z, z^{\prime}\right), \quad \text { on } \mathbb{R} \times \stackrel{\circ}{X} \times \stackrel{\circ}{X} \\
U(t)=0, \quad \text { if } t<0 .
\end{gathered}
$$

where $\delta\left(z, z^{\prime}\right)$ is the delta function with respect to the natural Riemannian measure given by the metric g. Theorem 1.1 shows that the limit of the Schwartz kernel

$$
\lim _{x \rightarrow 0} x^{-\frac{n-1}{2}} U\left(s+\frac{1}{x}, x, y, z^{\prime}\right)=\mathcal{R}_{+}\left(s, y, z^{\prime}\right)
$$

exists, but it does not give any information about what type of distribution it is.

We observe that, since the Lorentzian metric associated to $g$ is

$$
\sigma=d t^{2}-\frac{d x^{2}}{x^{4}}-\frac{h(x, y, d y)}{x^{2}}=d\left(t+\frac{1}{x}\right) d\left(t-\frac{1}{x}\right)-\frac{h(x, y, d y)}{x^{2}}
$$

the surfaces

$$
\left\{t-\frac{1}{x}=C\right\}, \quad\left\{t+\frac{1}{x}=C\right\}
$$

are characteristic for the wave operator, and thus a point $\left(t^{\prime}, z^{\prime}\right), z^{\prime}=\left(x^{\prime}, y^{\prime}\right)$, has a past domain of dependence, $\Delta^{-}\left(t^{\prime}, z^{\prime}\right)$ satisfying

$$
\Delta^{-}\left(t^{\prime}, z^{\prime}\right) \subset\left\{(t, x, y): t-\frac{1}{x} \leq t^{\prime}-\frac{1}{x^{\prime}}, t+\frac{1}{x} \leq t^{\prime}+\frac{1}{x^{\prime}}\right\} .
$$

\section{Radiation Fields And The Scattering Matrix}

For $w_{0}, w_{1} \in C_{0}^{\infty}(\stackrel{\circ}{X})$ the energy norm of $w=\left(w_{0}, w_{1}\right)$ is defined by

$$
\|w\|_{E}=\frac{1}{2} \int_{X}\left(\left|d w_{0}\right|_{g}^{2}+\left|w_{1}\right|^{2}\right) \operatorname{dvol}_{g}
$$

where $\left|d w_{0}\right|_{g}^{2}$ denotes the length of the co-vector with respect to the metric induced by $g$ on $T^{*} X$, and define $H_{E}(X)$ as the closure of $C_{0}^{\infty}(\stackrel{\circ}{X}) \times C_{0}^{\infty}(\stackrel{\circ}{X})$ with the norm $(2.1)$.

Let $W(t)$ be the map defined by

$$
\begin{gathered}
W(t): C_{0}^{\infty}(\stackrel{\circ}{X}) \times C_{0}^{\infty}(\stackrel{\circ}{X}) \longrightarrow C_{0}^{\infty}(\stackrel{\circ}{X}) \times C_{0}^{\infty}(\stackrel{\circ}{X}), \\
W(t)\left(f_{1}, f_{2}\right)=\left(u(t, z), D_{t} u(t, z)\right), \quad t \in \mathbb{R} .
\end{gathered}
$$

The conservation of energy gives, see for example the proof of Proposition 2.24 of [1], that

$$
W(t): H_{E}(X) \longrightarrow H_{E}(X), \quad t \in \mathbb{R},
$$

is a strongly continuous group of unitary operators.

Theorem 1.1 defines a map

$$
\begin{gathered}
\mathcal{R}_{+}: C_{0}^{\infty}(\stackrel{\circ}{X}) \times C_{0}^{\infty}(\stackrel{\circ}{X}) \longrightarrow C^{\infty}(\mathbb{R} \times \partial X) \\
\mathcal{R}_{+}\left(f_{1}, f_{2}\right)(s, y)=\left.\left(x^{-\frac{n-1}{2}} D_{t} H u\right)\left(s+\frac{1}{x}, x, y\right)\right|_{x=0}=D_{s} w_{0}(s, y),
\end{gathered}
$$

which will be called the forward radiation field.

Similarly one can prove that if $u_{-}$satisfies (1.5) and $H_{-}(t)=H(-t)$

$$
\lim _{x \rightarrow 0}\left(x^{-\frac{n-1}{2}} H_{-} u_{-}\right)\left(s-\frac{1}{x}, x, y\right)=w_{0}^{-}(s, y)
$$


exists, and thus define the backward radiation field as

$$
\begin{gathered}
\mathcal{R}_{-}: C_{0}^{\infty}(\stackrel{\circ}{X}) \times C_{0}^{\infty}(\stackrel{\circ}{X}) \longrightarrow C^{\infty}(\mathbb{R} \times \partial X) \\
\mathcal{R}_{-}\left(f_{1}, f_{2}\right)(s, y)=\left.\left(x^{-\frac{n-1}{2}} D_{t} H_{-} u_{-}\right)\left(s-\frac{1}{x}, x, y\right)\right|_{x=0}=D_{s} w_{0}^{-}(s, y) .
\end{gathered}
$$

By changing $t \leftrightarrow t-\tau$, the variable $s$ then changes to $s \leftrightarrow s+\tau$ therefore $\mathcal{R}_{ \pm}$satisfy

$$
\mathcal{R}_{ \pm} \circ(W(\tau) f)(y, s)=\mathcal{R}_{ \pm} f(y, s+\tau), \quad \tau \in \mathbb{R} .
$$

So Theorem 1.1 shows that $\mathcal{R}_{ \pm}$are "twisted" translation representations of the group $W(t)$. That is, if one sets $\widetilde{\mathcal{R}_{ \pm}}(f)(y, s)=\mathcal{R}_{ \pm} f(y,-s)$, then

$$
\widetilde{\mathcal{R}_{ \pm}}(W(\tau))=T_{\tau} \widetilde{\mathcal{R}_{ \pm}}
$$

where $T_{\tau}$ denotes the right translation by $\tau$ in the $s$ variable. So $\widetilde{\mathcal{R}_{ \pm}}$are translation representers in the sense of Lax and Phillips. We will use the results of $[1,2,3]$ to prove

Theorem 2.1. The maps $\mathcal{R}_{ \pm}$extend to isometries

$$
\mathcal{R}_{ \pm}: H_{E}(X) \longmapsto L^{2}(\partial X \times \mathbb{R}) .
$$

The scattering operator is defined to be the map

$$
\mathcal{S}=\mathcal{R}_{+} \circ \mathcal{R}_{-}^{-1}
$$

It is clearly a unitary in $L^{2}(\partial X \times \mathbb{R})$, and in view of (2.5), it commutes with translations in $s$. Therefore the Schwartz kernel $\mathcal{S}\left(s, y, s^{\prime}, y^{\prime}\right)$ of $\mathcal{S}$ is completely determined by its values at $s=s^{\prime}$. In fact it satisfies

$$
\mathcal{S}\left(s, y, s^{\prime}, y^{\prime}\right)=\mathcal{S}\left(\frac{s+s^{\prime}}{2}, y, \frac{s+s^{\prime}}{2}, y^{\prime}\right) \text {. }
$$

The scattering matrix is defined by conjugating $\mathcal{S}$ with the partial Fourier transform in the $s$ variable

$$
\mathcal{A}=\mathcal{F} \mathcal{S F}^{-1} \text {. }
$$

$\mathcal{A}$ is a unitary operator in $L^{2}(\partial X \times \mathbb{R})$. Since $\mathcal{S}$ acts as a convolution in the variable $s^{\prime}$, if $\lambda$ denotes the dual variable to $s, \mathcal{A F}$ is a multiplication in $\lambda$, i.e

$$
\mathcal{A}(\mathcal{F} F)(\lambda, y)=\int_{\partial X} \mathcal{A}\left(\lambda, y, y^{\prime}\right) \mathcal{F} F\left(\lambda, y^{\prime}\right) \operatorname{dvol}_{h}
$$

It is a consequence of the results of section 9 of [3] that the stationary and dynamical definitions of the scattering matrix coincide. More precisely:

Theorem 2.2. The Schwartz kernel of the map $A(\lambda)$ defined by (1.4) is equal to $\mathcal{A}\left(\lambda, y, y^{\prime}\right)$.

\section{Proofs of Theorems 2.1 And 2.2}

Friedlander proved that $\mathcal{R}_{+}$is a partial isometry. We put together the results from Lemma 3.22, Proposition 3.24 and Theorem 3.25 of [1] in

Theorem 3.1. ([1]) The space

$$
H_{E}^{1}=\left\{f \in H_{E}:\left\|\mathcal{R}_{+} f\right\|_{L^{2}(\mathbb{R} \times \partial X)}=\|f\|_{H_{E}(X)}\right\}
$$

is a closed subspace of $H_{E}$, and for $f \in H_{E}$ and $g \in H_{E}^{1}$,

$$
\langle f, g\rangle_{H_{E}}=\left\langle\mathcal{R}_{+} f, \mathcal{R}_{+} g\right\rangle_{L^{2}(\mathcal{R} \times \partial X)}
$$

Moreover

$$
\mathcal{R}_{+}: H_{E}^{1} \longrightarrow L^{2}(\mathbb{R} \times \partial X)
$$


is a surjective isometry and

$$
H_{E}=H_{E}^{1} \oplus \operatorname{Ker}\left(\mathcal{R}_{+}\right), \quad \operatorname{Ker}\left(\mathcal{R}_{+}\right)=\left\{f \in H_{E}: \mathcal{R}_{+} f=0\right\}, .
$$

These results were proved in [1] for a metric perturbation of the Euclidean metric, but in fact no intrinsic properties of $\mathbb{R}^{n}$ were used and, as Friedlander pointed out in the paragraph after equation (44) of [2], they can be easily extended to the asymptotically Euclidean case. Actually his approach in [2] can be used to simplify the proofs in [1].

We will show that Theorem 2.1 follows from the results of [3].

Proof. To see this, first observe that if $u(t, z)$ satisfies (1.5), then

$$
\left(D_{t}^{2}-\Delta\right)(H u)(t, z)=i f_{2}(z) \delta(t)-i f_{1}(z) D_{t} \delta(t) .
$$

Taking partial Fourier-Laplace transform in $t$ gives

$$
\left(\Delta-\lambda^{2}\right) \widehat{(H u)}(\lambda, z)=i \lambda f_{1}(z)-i f_{2}(z), \quad \Im \lambda<0
$$

So, in terms of the resolvent $R(\lambda)=\left(\Delta-\lambda^{2}\right)^{-1}$,

$$
\widehat{(H u)}(\lambda, z)=R(\lambda)\left(i \lambda f_{1}-i f_{2}\right)(z), \Im \lambda<0 .
$$

On the other hand, we observe that the Fourier transform in $s$ of $v_{+}(x, s, y)=x^{-\frac{n-1}{2}}(H u)\left(s+\frac{1}{x}, x, y\right)$ is

$$
\widehat{v_{+}}(x, \lambda, y)=i x^{-\frac{n-1}{2}} e^{i \frac{\lambda}{x}} R(\lambda)\left(\lambda f_{1}-f_{2}\right)(x, y), \quad \Im \lambda<0 .
$$

Similarly, if $v_{-}(x, s, y)=x^{-\frac{n-1}{2}}\left(H_{-} u_{-}\right)\left(s-\frac{1}{x}, x, y\right)$

$$
\widehat{v_{-}}(x, \lambda, y)=i x^{-\frac{n-1}{2}} e^{-i \frac{\lambda}{x}} R(\lambda)\left(-i \lambda f_{1}+f_{2}\right)(x, y), \quad \Im \lambda>0 .
$$

From Theorem 3.1 we know that

$$
\lambda \widehat{w_{0}}(\lambda, y)=\lambda \widehat{v_{+}}(0, \lambda, y), \in L^{2}(\mathbb{R} \times \partial X) \quad \text { and } \quad \lambda \widehat{w_{0}^{-}}(\lambda, y)=\lambda \widehat{v_{-}}(0, \lambda, y) \in L^{2}(\mathbb{R} \times \partial X) .
$$

As shown in the proof of Lemma 5.2 of [3],

$$
\lim _{x \downarrow 0, \Im \lambda \uparrow 0} x^{-\frac{n-1}{2}} e^{i \frac{\lambda}{x}} R(\lambda)=-\frac{i}{2 \lambda} P(\lambda)^{*},
$$

where $P(\lambda)^{*}$ is the adjoint of the Poisson operator $P(\lambda)$, which is the map that takes $f \in C^{\infty}(\partial X)$ into the solution to (1.3). Therefore the limits of (3.1) and (3.2) are

$$
\begin{aligned}
\lambda \widehat{w_{0}}(\lambda, y) & =\frac{1}{2} P(\lambda)^{*}\left(\lambda f_{1}-f_{2}\right), \\
\widehat{w_{0}^{-}}(\lambda, y) & =\frac{1}{2} P(-\lambda)^{*}\left(\lambda f_{1}-f_{2}\right) .
\end{aligned}
$$

and hence

$$
\begin{aligned}
\mathcal{F}\left(\mathcal{R}_{+}\left(f_{1}, f_{2}\right)\right)(\lambda, y) & =\frac{1}{2} P(\lambda)^{*}\left(\lambda f_{1}-f_{2}\right), \\
\mathcal{F}\left(\mathcal{R}_{-}\left(f_{1}, f_{2}\right)\right)(\lambda, y) & =\frac{1}{2} P(-\lambda)^{*}\left(\lambda f_{1}-f_{2}\right) .
\end{aligned}
$$

Next we appeal to the results of section 9 of [3]. As we have no discrete spectrum, it follows from Proposition 9.1 of [3], and the fact that $P( \pm \lambda)^{*} \Delta=\lambda^{2} P( \pm \lambda)^{*}$, that

$$
\begin{gathered}
\left\|\mathcal{F}\left(\mathcal{R}_{+}\left(f_{1}, f_{2}\right)\right)\right\|_{L^{2}(\mathbb{R} \times \partial X)}^{2}=\frac{1}{2} \int_{0}^{\infty}\left|P(\lambda)^{*} f_{2}\right|^{2} d \lambda+\frac{1}{2} \int_{0}^{\infty}\left|P(\lambda)^{*} \lambda^{2} f_{1}\right|^{2} d \lambda= \\
\pi\left\|f_{2}\right\|_{L^{2}(X)}^{2}+\pi\left\langle f_{1}, \Delta f_{1}\right\rangle_{L^{2}(X)}=2 \pi\left\|\left(f_{1}, f_{2}\right)\right\|_{E}^{2} .
\end{gathered}
$$


One should notice that the integral on the left hand side is taken over $\mathbb{R}$, and the cross terms involving $f_{1}$ and $f_{2}$ are odd, so their integral is zero. This proves Theorem 2.1.

It is also proved in section 9 of [3] that the scattering matrix $A(\lambda)$ is the unitary operator on $L^{2}(\partial X)$ that intertwines these operators, i.e

$$
A(\lambda) P(-\lambda)^{*}=P(\lambda)^{*}
$$

Hence from (3.3)

$$
A(\lambda) \mathcal{F} \mathcal{R}_{-}=\mathcal{F} \mathcal{R}_{+}
$$

This gives Theorem 2.2.

\section{A SUPPORT THEOREM FOR $\mathcal{R}_{+}$}

The proof relies on Hörmander's uniqueness theorem for the Cauchy problem across a strongly pseudoconvex surface and the following uniqueness theorem, which is a particular case of a result due to Tataru $[14]$, see also $[11,12,15]$.

Theorem 4.1. ([14]) Let $g$ be a smooth Riemannian metric on a smooth manifold and let $\Delta_{g}$ its Laplacian. Let $L$ be a first order smooth operator independent of $t$ and let $u(t, z) \in C^{\infty}(\mathbb{R} \times X)$ satisfy

$$
\begin{gathered}
\left(D_{t}^{2}-\Delta_{g}-L\right) u(t, z)=0 \\
u(t, z)=0 \quad \forall(t, z) \in[-T, T] \times\left\{z ; \quad d\left(z, z_{0}\right)<\delta\right\}, \quad \delta>0 .
\end{gathered}
$$

Then $u(t, z)=0$ for $|t|+d\left(z, z_{0}\right)<T$, where $d\left(z, z_{0}\right)$ denotes the distance between $z$ and $z_{0}$ with respect to $g$.

Lemma 4.1. Let $(f, g) \in C_{0}^{\infty}(\stackrel{\circ}{X})$ be supported in $\left\{z=(x, y): x>x_{0}\right\}$. Let $u(t, x, y)$ be the solution of (1.5). If $0>s_{0}>-\frac{1}{x_{0}}$ and $\mathcal{R}_{+}(f, g)(s, y)=0$ for $s<s_{0}$, then $u(t, x, y)=0$ for $0 \leq x \leq x_{0}$ and $0 \leq t \leq s_{0}+\frac{1}{x_{0}}$.

Proof. We will show that if

$$
v(x, s, y)=x^{-\frac{n-1}{2}} u\left(s+\frac{1}{x}, x, y\right)
$$

satisfies $\frac{\partial}{\partial s} v(0, s, y)=0$ for $s<s_{0}$, then

$$
v(x, s, y)=0 \text { in the set }\left\{(x, s, y): 0 \leq x \leq x_{0},-\frac{1}{x_{0}}<s<s_{0}\right\} .
$$

Translating this back to the variables $(x, y, t)$, and using finite speed of propagation, this gives the statement of the Lemma.

Let $P=x^{-2-\frac{n-1}{2}}\left(D_{t}^{2}-\Delta\right) x^{\frac{n-1}{2}}$. Taking $s=t-\frac{1}{x}$, it follows that

$$
P v=0 \text { and }
$$

$$
P=2 \frac{\partial}{\partial x} \frac{\partial}{\partial s}+x^{2} \frac{\partial^{2}}{\partial x^{2}}+\Delta_{h}+A \frac{\partial}{\partial s}+\left(2 x+x^{2} A\right) \frac{\partial}{\partial x}+\left(\frac{n-1}{2}\right)\left(\frac{3-n}{2}+x A\right),
$$

Here $A(x, y)=\frac{\partial}{\partial x} \log \sqrt{|h|}$ and $\Delta_{h}$ is the (negative) Laplacian with respect to the metric $h$, i.e $\Delta_{h}=$ $\frac{1}{\sqrt{h}} \frac{\partial}{\partial y_{i}} h^{i j} \sqrt{h} \frac{\partial}{\partial y_{j}}$.

By finite speed of propagation,

$$
v(x, s, y)=0 \text { in }\left\{-\frac{2}{x}+\frac{1}{x_{0}}<s<-\frac{1}{x_{0}}\right\},
$$


We also know that $v$ is smooth up to $x=0$. So let

$$
\frac{\partial}{\partial s} v(x, s, y) \sim \sum_{k=0}^{\infty} v_{k}(s, y) x^{k}, \quad \text { as } \quad x \rightarrow 0
$$

denote its Taylor series at $x=0$. From (4.3)

$$
v_{k}(s, y)=0, \quad s<-\frac{1}{x_{0}}
$$

By assumption $\frac{\partial}{\partial s} v(0, s, y)=0$ for $s<s_{0}$. So

$$
v_{0}(s, y)=0 \text { for } s<s_{0} \text {. }
$$

Let

$$
\begin{gathered}
Q=\Delta_{h}+\left(\frac{n-1}{2}\right)\left(\frac{3-n}{2}\right) \\
Q=Q_{0}+x Q_{1}+x^{2} Q_{2}+\ldots \\
A=A_{0}+x A_{1}+x^{2} A_{2}+\ldots
\end{gathered}
$$

Then

$$
\begin{gathered}
0 \sim 2 \frac{\partial}{\partial s} v_{1}+\left(A_{0} \frac{\partial}{\partial s}+Q_{0}\right) v_{0}+\left(4 \frac{\partial}{\partial s} v_{2}+\left(2+Q_{0}+A_{0} \frac{\partial}{\partial s}\right) v_{1}+\left(Q_{1}+A_{1} \frac{\partial}{\partial s}+A_{0}\right) v_{0}\right) x+ \\
+\sum_{k=2}^{\infty}\left[2(k+1) \frac{\partial}{\partial s} v_{k+1}+k(k+1) v_{k}+\sum_{j+m=k}\left(Q_{j} v_{m}+A_{j} \frac{\partial}{\partial s} v_{m}\right)+\sum_{j+m=k-1}\left(A_{j} v_{m}+m A_{j} v_{m}\right)\right] x^{k} \\
v_{k}=0 \text { for } s<-\frac{1}{x_{0}}, \quad k=0,1, \ldots
\end{gathered}
$$

Since $v_{0}(s, y)=0$ for $s<s_{0}$, then it follows that $\frac{\partial}{\partial s} v_{1}(s, y)=0$ for $s<s_{0}$. From (4.4), $v_{1}(s, y)=0$ for $s<s_{0}$. Proceeding the same way we find that $v_{k}(s, y)=0$ for $s<s_{0}$ and $k \in \mathbb{N}$. So $v$ vanishes to infinite order at $\left\{x=0, s<s_{0}\right\}$ and $\left\{s=-\frac{1}{x_{0}}, x<\epsilon_{0}\right\}$, and therefore we can extend it as a solution to (4.2) in a neighborhood of $\left\{s=-\frac{1}{x_{0}}, \quad x=0\right\}$, which is equal to zero in $x<0$. More precisely the extension of $v$ vanishes in $\left\{x<0, s<s_{0}\right\}$, see figure 1 .

Let $\phi(x, s)=-x-\delta\left(s+\frac{1}{x_{0}}\right)+\left(s+\frac{1}{x_{0}}\right)^{2}$. For $\delta>0$ small enough, this function is strongly pseudoconvex with respect to $P$ at every point of the set $\Sigma=\left\{x=0, s=-\frac{1}{x_{0}}\right\}$. Indeed, as $P$ is of second order, and the level surface $\{\phi=0\}$ is non-characteristic at $\Sigma$, then, according to the equation after the first paragraph in section 28.4 of [5], strong pseudoconvexity reduces to the condition

$$
\left(H_{p}^{2} \phi\right)\left(0,-\frac{1}{x_{0}}, y, \xi, \sigma, \eta\right)>0, \text { if } \quad p\left(0,-\frac{1}{x_{0}}, y, \xi, \sigma, \eta\right)=\left(H_{p} \phi\right)\left(0,-\frac{1}{x_{0}}, y, \xi, \sigma, \eta\right)=0, \quad(\xi, \sigma, \eta) \neq 0,
$$

where $p$ is the principal symbol of $P$ and $H_{p}$ its Hamiltonian. In this case

$$
\begin{gathered}
p=-2 \xi \sigma-x^{2} \xi^{2}-h(x, y, \eta), \quad \text { and } \\
H_{p}=-2\left(\sigma+x^{2} \xi\right) \frac{\partial}{\partial x}-2 \xi \frac{\partial}{\partial s}+\left(2 x \xi^{2}+\frac{\partial h}{\partial x}\right) \frac{\partial}{\partial \xi}-H_{h}
\end{gathered}
$$


where $h=\sum_{i, j} h^{i j} \eta_{i} \eta_{j}$ is the principal symbol of $\Delta_{h}$, and $H_{h}$ denotes the Hamiltonian of $h$, which only includes derivatives in $y$ and $\eta$. So

$$
\begin{gathered}
p\left(0,-\frac{1}{x_{0}}, y, \xi, \sigma, \eta\right)=-2 \xi \sigma-h(0, y, \eta), \quad\left(H_{p} \phi\right)\left(0,-\frac{1}{x_{0}}, y, \xi, \sigma, \eta\right)=2 \sigma+2 \delta \xi \\
\left(H_{p}^{2} \phi\right)\left(0,-\frac{1}{x_{0}}, y, \xi, \sigma, \eta\right)=8 \xi^{2}+2 \delta \frac{\partial h}{\partial x}(0, y, \eta) . \\
y, \xi, \sigma, \eta)=\left(H_{p} \phi\right)\left(0,-\frac{1}{x_{0}}, y, \xi, \sigma, \eta\right)=0, \text { then } \\
\sigma=-\delta \xi, h(0, y, \eta)=2 \delta \xi^{2}, \text { and } \\
H_{p}^{2} \phi\left(0,-\frac{1}{x_{0}}, \xi, \sigma, \eta\right)=\frac{2}{\delta}\left(2 h(0, y, \eta)+\delta^{2} \frac{\partial h}{\partial x}(0, y, \eta)\right) .
\end{gathered}
$$

If $p\left(0,-\frac{1}{x_{0}}, y, \xi, \sigma, \eta\right)=\left(H_{p} \phi\right)\left(0,-\frac{1}{x_{0}}, y, \xi, \sigma, \eta\right)=0$, then

As $h(0, y, \eta)$ is positive definite, $\delta$ can be chosen so that

$$
H_{p}^{2} \phi\left(0,-\frac{1}{x_{0}}, \xi, \sigma, \eta\right) \geq C|\eta|^{2}, \quad \eta \neq 0 .
$$

Thus, for this choice of $\delta,(4.5)$ is satisfied.

Since $v=0$ in $\{(x, s, y): \phi(x, s)>0\}$, for $x, s$ small enough, Hörmander's uniqueness theorem, Theorem 28.3.4 of [5], implies that $v=0$ in a neighborhood of $\left\{x=0, s=-\frac{1}{x_{0}}\right\}$. Therefore, there exists $\epsilon>0$ such that

$$
v(x, s, y)=0 \quad \text { if } \quad-\epsilon<x<\epsilon,-\frac{1}{x_{0}}-\epsilon<s<-\frac{1}{x_{0}}+\epsilon
$$

Now we apply the same argument used above to show that $v(x, s, y)=0$ in a neighborhood of $\{x=$ $\left.0, s=-\frac{1}{x_{0}}+\epsilon\right\}$. Using the compactness of $\left[-\frac{1}{x_{0}}, s_{0}\right]$, the argument above can be repeated a finite number of times to show that there exists $\epsilon_{0}>0$ such that

$$
v(x, s, y)=0 \quad \text { if } \quad-\epsilon_{0}<x<\epsilon_{0},-\frac{2}{x}+\frac{1}{x_{0}}<s<s_{0} .
$$

Given $x_{1} \in\left(0, x_{0}\right)$, we can use a compactness argument and the same method as above to show that

$$
v(x, s, y)=0 \quad \text { if } 0<x<x_{1},-\frac{2}{x}+\frac{1}{x_{0}}<s<s_{0} .
$$

The smoothness of $v$ then guarantees that

$$
v(x, s, y)=0 \quad \text { if } \quad 0<x \leq x_{0}, \quad-\frac{1}{x_{0}}<s<s_{0} .
$$

See figure 1.

The following is the main result of this section:

Theorem 4.2. Let $f \in C_{0}^{\infty}(\stackrel{\circ}{X})$ and let $s_{0}<0$. If $\mathcal{R}(0, f)(s, y)=0$ (or $\mathcal{R}(f, 0)=0$ ) for all $s<s_{0}$ then $f=0$ in the set $\left\{(x, y) \in X: x<-\frac{1}{s_{0}}\right\}$.

Proof. Since $f$ is compactly supported in the interior of $X$, there exists $x_{0}$ such that $f(x, y)=0$ for $x<x_{0}$. If $x_{0}>-\frac{1}{s_{0}}$, there is nothing to be proved. So we assume that $x_{0}<-\frac{1}{s_{0}}$. It follows from Lemma 4.1, and the observation that $u(t, z)$ is odd in $t$, that $u(t, z)=0$ for $x<x_{0}$ and $-s_{0}-\frac{1}{x_{0}}<t<$ $s_{0}+\frac{1}{x_{0}}$. Thus it follows from Theorem 4.1 that for $X_{x_{0}}=\left\{\left(x_{0}, y\right)\right\}, u(0, z)=D_{t} u(0, z)=0$ in the set $\left\{z: d\left(z, X_{x_{0}}\right)<s_{0}+\frac{1}{x_{0}}\right\}$. In this metric this distance is

$$
d\left(z, X_{x_{0}}\right)=\int_{x_{0}}^{x(z)} \frac{1}{\tau^{2}} d \tau=\frac{1}{x_{0}}-\frac{1}{x(z)} .
$$

Thus if $d\left(z, X_{x_{0}}\right)<s_{0}+\frac{1}{x_{0}}$ it follows that $x(z)<-\frac{1}{s_{0}}$. 


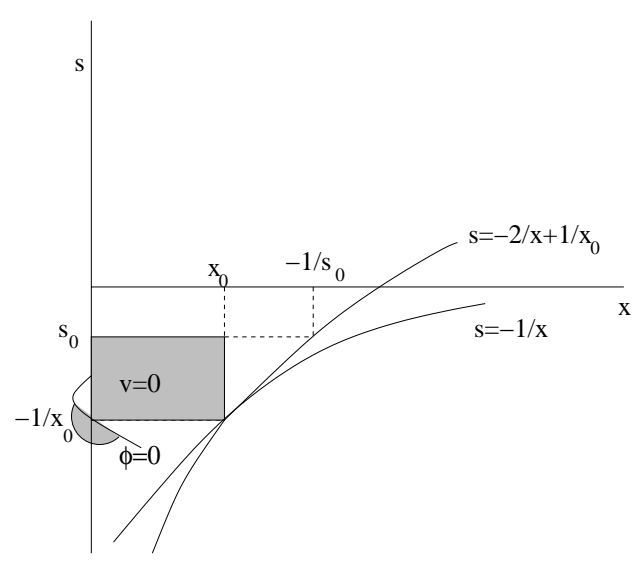

FiguRE 1. Region of uniqueness.

Theorem 2.4 in chapter 4 of [7], see also Proposition 4.20 of [1], states that, in the odd dimensional Euclidean space $\mathbb{R}^{n}$,

$$
\mathcal{R}_{+}(0, f)(s, \omega)=\left(\frac{\partial}{\partial s}\right)^{\frac{n-1}{2}} R f(s, \omega)
$$

where

$$
R f(s, \omega)=\int_{\langle x, \omega\rangle=s} f(x) d H_{x}
$$

is the Radon transform. Thus, in this particular case, Theorem 4.2 is the equivalent of the support theorem for Radon transform, see [4], which says that if $f \in C_{0}^{\infty}\left(\mathbb{R}^{n}\right)$ and $R f(s, \omega)=0$ for $|s|>\rho$, then $f(z)=0$ for $|z|>\rho$. However, the proof given here is by no means a simplification of Helgason's proof.

\section{Acknowledgments}

I am grateful for the financial support I received from the NSF under grant DMS 0140657. I thank the two referees for carefully reading the first version of this paper and for making numerous suggestions and corrections.

\section{REFERENCES}

[1] F.G. Friedlander. Radiation fields and hyperbolic scattering theory. Math. Proc. Camb. Phil. Soc., 88, 483-515, (1980).

[2] F.G. Friedlander. Notes on the wave equation on asymptotically Euclidean manifolds. J. of Func. Anal. 184, no. 1, $1-18,(2001)$.

[3] A. Hassell and A. Vasy. The spectral projections and the resolvent for scattering metrics. J. Anal. Math. $79241-298$. (1999).

[4] S. Helgason. The Radon Transform. Birkhauser, 2nd edition, (1999).

[5] L. Hörmander. The analysis of linear partial differential operators IV. Springer-Verlag, 1985.

[6] M.S. Joshi and A. Sá Barreto. Recovering asymptotics of metrics from fixed energy scattering data. Invent. Math. 137, 127-143, (1999)

[7] P. Lax and R. Phillips. Scattering theory. Academic Press, 1989, Revised edition .

[8] R.B. Melrose. Geometric scattering theory. Cambridge Univ. Press, 1995.

[9] R.B. Melrose. Spectral and scattering theory for the Laplacian on asymptotically Euclidean spaces. Spectral and Scattering Theory. (M. Ikawa, ed.) Marcel Dekker, 1994.

[10] R.B. Melrose and M. Zworski. Scattering metrics and geodesic flow at infinity. Invent. Math. 124, 389-436 (1996).

[11] L. Robbiano. Théorème d'unicité adapté au contrôle des solutions des problémes hyperboliques. Comm. in P.D.E 16, 789-800, (1991). 
[12] L. Robbiano and C. Zuilly. Uniqueness in the Cauchy problem for operators with partially holomorphic coefficients. Invent. Math. 131, 493-539, (1998).

[13] A. Sá Barreto. Radiation fields and inverse scattering on asymptotically hyperbolic manifolds. Preprint, 2002.

[14] D. Tataru. Unique continuation for solutions to PDE's: between Hörmander's theorem and Holmgren's theorem. Comm. in P.D.E. 20, 855-884, (1995).

[15] D. Tataru. Unique continuation for operators with partially analytic coefficients. J. Math. Pures Appl. 78, 505-521, 1999.

Department of Mathematics Purdue University, West Lafayette IN 47907, U.S.A.

E-mail address: sabarre@math.purdue.edu 